\title{
The ARASE (ERG) magnetic field investigation
}

\author{
Ayako Matsuoka ${ }^{1 *} \mathbb{D}$, Mariko Teramoto ${ }^{2}$, Reiko Nomura ${ }^{3}$, Masahito Nosé ${ }^{4}$, Akiko Fujimoto ${ }^{5}$, Yoshimasa Tanaka ${ }^{6}$, \\ Manabu Shinohara ${ }^{7}$, Tsutomu Nagatsuma ${ }^{8}$, Kazuo Shiokawa ${ }^{2}$, Yuki Obana ${ }^{9}$, Yoshizumi Miyoshi ${ }^{2}$, Makoto Mita', \\ Takeshi Takashima' and Iku Shinohara'
}

\begin{abstract}
The fluxgate magnetometer for the Arase (ERG) spacecraft mission was built to investigate particle acceleration processes in the inner magnetosphere. Precise measurements of the field intensity and direction are essential in studying the motion of particles, the properties of waves interacting with the particles, and magnetic field variations induced by electric currents. By observing temporal field variations, we will more deeply understand magnetohydrodynamic and electromagnetic ion-cyclotron waves in the ultra-low-frequency range, which can cause production and loss of relativistic electrons and ring-current particles. The hardware and software designs of the Magnetic Field Experiment (MGF) were optimized to meet the requirements for studying these phenomena. The MGF makes measurements at a sampling rate of 256 vectors $/ \mathrm{s}$, and the data are averaged onboard to fit the telemetry budget. The magnetometer switches the dynamic range between \pm 8000 and $\pm 60,000 \mathrm{nT}$, depending on the local magnetic field intensity. The experiment is calibrated by preflight tests and through analysis of in-orbit data. MGF data are edited into files with a common data file format, archived on a data server, and made available to the science community. Magnetic field observation by the MGF will significantly improve our knowledge of the growth and decay of radiation belts and ring currents, as well as the dynamics of geospace storms.
\end{abstract}

Keywords: EMIC wave, Geospace, Magnetic field, Magnetometer, Radiation belts, Ring current, ULF wave

\section{Introduction}

The Arase (also known as Exploration of energization and Radiation in Geospace, ERG) satellite was successfully launched on December 20, 2016, from the Uchinoura Space Center. The spacecraft has apogee and perigee altitudes of $\sim 32,000$ and $\sim 440 \mathrm{~km}$, respectively, and an inclination of $32^{\circ}$, allowing the spacecraft to spend a majority of its time in the radiation belts. The spacecraft has an orbital period of $570 \mathrm{~min}$ and is spin-stabilized with a spin period of $\sim 8 \mathrm{~s}$.

The primary objective of the Arase mission is to reveal the generation mechanisms of relativistic electrons in radiation belts (Miyoshi et al. 2013; Miyoshi

\footnotetext{
*Correspondence: matsuoka@isas.jaxa.jp

${ }^{1}$ Institute of Space and Astronautical Science, Japan Aerospace

Exploration Agency, 3-1-1, Yoshinodai, Chuo-ku, Sagamihara, Kanagawa

252-5210, Japan

Full list of author information is available at the end of the article
}

et al. 2017). Particles of various energies and waves of various frequencies are considered to be involved in the electron acceleration process in the inner magnetosphere. The Arase spacecraft measures the fluxes of ions and electrons over a wide energy range and detects electric and magnetic field oscillations over a wide frequency range to gain insight into the interactions between particles of different energies and waves of different frequencies.

The magnetic field is a fundamental quantity in space plasma studies. The behavior of charged particles is often examined in a coordinate system referenced to the direction of the magnetic field. Moreover, temporal variations in the magnetic field play an important role in the transport, acceleration, and deceleration process of charged particles. The Magnetic Field Experiment (MGF) for the Arase mission was developed to conduct precise measurements of the static magnetic field and low-frequency magnetic field variations. 
In "Science objectives and requirements for MGF" section of this of paper, we describe the scientific objectives of the MGF and its requirements. In "Instrument design", "Performance of MGF", and "Spacecraft magnetic cleanliness" sections, we explain the instrument design, performance, and the magnetic cleanliness of the spacecraft, respectively. The compliance of the MGF to meet specification is discussed in each section. In "Operation" and "In-orbit calibration" sections, we describe the in-orbit operation of MGF and data calibration based on preflight experiments and in-orbit data analysis, respectively. In "Data processing flow" section, we show the MGF data processing flow for the scientific studies.

\section{Science objectives and requirements for MGF Background field strength and direction}

In the region of geospace surveyed by Arase, the magnetic field is dominated by the component originating from the earth's interior; therefore, the field is well represented by the International Geomagnetic Reference Field (IGRF) model. Meanwhile, global electric currents flowing in the magnetosphere cause deviations from the intrinsic field, and temporal variations in the electric current in the magnetosphere produce temporal variations in the magnetic field configuration.

Cummings et al. (1968) first reported that during magnetospheric substorms, a tailward-stretched magnetic field recovers rapidly to a dipolar configuration, a phenomenon known as dipolarization. Magnetic dipolarization has often been discussed in association with bursty bulk flow (BBF) in the magnetotail (e.g., Angelopoulos et al. 1992; Takada et al. 2006). According to previous studies, particle acceleration occurs during dipolarization, although the causal relationship between the particle and magnetic field behaviors and the physical process connecting them are still under study (Lui 1991; McPherron and Chu 2016). In the inner magnetosphere, a change in the field configuration can induce particle acceleration (Delcourt 2002). Understanding the relationship between variation in the field configuration and the generation of energetic particles is one of the major objectives of the Arase project.

Observing the pitch-angle distribution of charged particles is essential in studying wave-particle interactions because an anisotropic distribution contains free energy to excite plasma waves. Accurate determination of the field direction is necessary to study the dependence of the particle characteristics on the pitch angle. By measuring the pitch-angle distribution of the particle flux, we may gain information on phenomena occurring at varying distances from the spacecraft along the field line. For example, particle dissipation at low altitudes could produce a loss cone distribution observed on the same field line in the equatorial region (e.g., Thorne et al. 2010).

\section{Pc3-5 ultra-low-frequency waves}

Low-frequency magnetic field variations observed in the inner magnetosphere are categorized according to frequency and waveform characteristics. Narrow-band waves having a period of several tens to a few $100 \mathrm{~s}$ are called Pc3-Pc5 ultra-low-frequency (ULF) waves. ULF waves have the potential to energize particles (Elkington et al. 1999; Liu et al. 1999; Shprits et al. 2008 and references therein). Charged particles in the inner magnetosphere basically have stable trajectories around the earth to keep the third adiabatic invariant constant. Meanwhile, acceleration could occur if particles periodically scattered by magnetic perturbations undergo radial diffusion.

Until the 1990s, most observations in the inner magnetosphere were made at geosynchronous orbit. The first comprehensive studies of ULF waves inward of the geostationary orbit were made using observations by the Active Magnetospheric Particle Tracer Explorers (AMPTE)/Charge Composition Explorer (CCE) at 5-8 $R_{\mathrm{E}}$ ( $R_{\mathrm{E}}$ is the radius of the earth) (Anderson et al. 1990; Anderson 1994). Ali et al. (2015) investigated ULF waves observed by the Combined Release and Radiation Effects Satellite (CRRES) at $L=4-6.5$ and evaluated the radial diffusion coefficient. The contribution of ULF waves to relativistic electron generation is still controversial; $\mathrm{Su}$ et al. (2015) concluded that radial diffusion plays a key role, whereas a substantial number of studies support gyroresonant electron-whistler mode wave interaction as the predominant process (Miyoshi et al. 2003; Horne et al. 2005a, b; Shprits et al. 2008; Reeves et al. 2013 and references therein).

\section{Electromagnetic ion-cyclotron waves}

It is proposed that the ions having a strong anisotropic pitch-angle distribution excite electromagnetic ioncyclotron (EMIC) waves in the Pc1-2 band (from 0.1 to several $\mathrm{Hz}$ ).

The statistics of the EMIC wave intensity observed by AMPTE/CCE were given by Anderson et al. (1992a), and the spatial distribution was demonstrated by Anderson et al. (1992b). The spatial occurrence frequency was further studied by Min et al. (2012) using Time History of Events and Macroscale Interactions during Substorms (THEMIS) data and by Keika et al. (2013) using AMPTE/ CCE data.

Studies that compare observation with numerical models suggest that EMIC waves contribute to the loss of energetic ions (Jordanova et al. 2003, 2006) and relativistic electrons (Summers and Thorne 2003; Miyoshi et al. 2008; Kersten et al. 2014). Nomura et al. (2012, 2016) reported proton aurora events that were supposedly caused by EMIC wave scattering. Sakaguchi et al. (2013) 
showed an EMIC rising tone event observed by Akebono in the deep inner magnetosphere and suggested the precipitation of relativistic electrons via EMIC waves.

\section{Requirements for the MGF}

Magnetic field data are necessary not only for the reasons mentioned above but also for almost all scientific studies by Arase.

To capture phenomena that Arase should encounter, we defined the following requirements for MGF capability and performance: temporal variation noise level lower than $80 \mathrm{pT} / \sqrt{\mathrm{Hz}}$ in the $0.1-10 \mathrm{~Hz}$ frequency range, field intensity error lower than $5 \mathrm{nT}$, and field direction error lower than $1^{\circ}$. These requirements apply to observations at $L>2\left(L\right.$ in $R_{\mathrm{E}}$ is the distance of the field line from the earth center at the magnetic equator). We designed and tested the MGF hardware, software, and spacecraft system to meet these criteria. Sensor noise is described in "MGF performance" section, spacecraft noise in "Spacecraft magnetic cleanliness" section, and the conformity of the MGF to the second and third criteria in "In-orbit calibration" section.

\section{Instrument design}

\section{Overview}

The MGF is a fluxgate magnetometer designed based on the magnetometer (MGF-I) developed for the BepiColombo mission's Mercury Magnetospheric Orbiter (MMO) (Baumjohann et al. 2006, 2010). The analogtype magnetometer follows the basic design adopted for many space missions (Gordon and Brown 1972; Fukunishi et al. 1990; Kokubun et al. 1994; Yamamoto and Matsuoka 1998; Tsunakawa et al. 2010). Nevertheless, some elements of the sensor and electronics were newly developed to withstand the harsh radiation environment of Mercury (Matsuoka et al. 2013). These elements are appropriate for the Arase spacecraft, which is also exposed to severe radiation.

Figure 1 shows the overall configuration of the MGF. It consists of a sensor (MGF-S) and an electronics box (MGF-E). The MGF-E is installed inside the spacecraft body, on the $-Z$ panel, where the $-Z$-direction roughly points toward the sun. It includes four electronics boards: the magnetometer circuit, the sensor temperature measurement circuit, the central processing unit (CPU), and the power supply unit (PSU). The PSU supplies $\pm 7 \mathrm{~V}$ power to the magnetometer circuit board and $+3.3 \mathrm{~V}$ to the CPU. Commands from the ground or issued autonomously by the spacecraft system are transferred to the CPU from the mission data processor (MDP) by Spacewire protocol. The CPU exchanges the commands and data with the magnetometer circuit board by the Spacewire protocol as well. Two types of data are generated by the magnetometer circuit board: mission (magnetic field) data and housekeeping (HK) data. Mission and HK telemetry packets are created by the CPU and sent to the ground via the MDP. Meanwhile, 'shared' magnetic field data are distributed to other instruments onboard Arase. In parallel, near-real-time magnetic field data are compiled with data from the extremely high-energy electron experiments (XEP) instrument and high-energy electron experiments (HEP) instrument in space-weather telemetry packets, and downlinked immediately for nearreal-time monitoring of the inner magnetosphere. The production/processing procedure of magnetic field data is described in "Onboard data processing" section.

The sensor (MGF-S) is mounted at the tip of an extendable 5-m MAST. The cables connecting MGF-S with MGF-E are twist-pair with a shield, which is electrically connected to the spacecraft ground level.

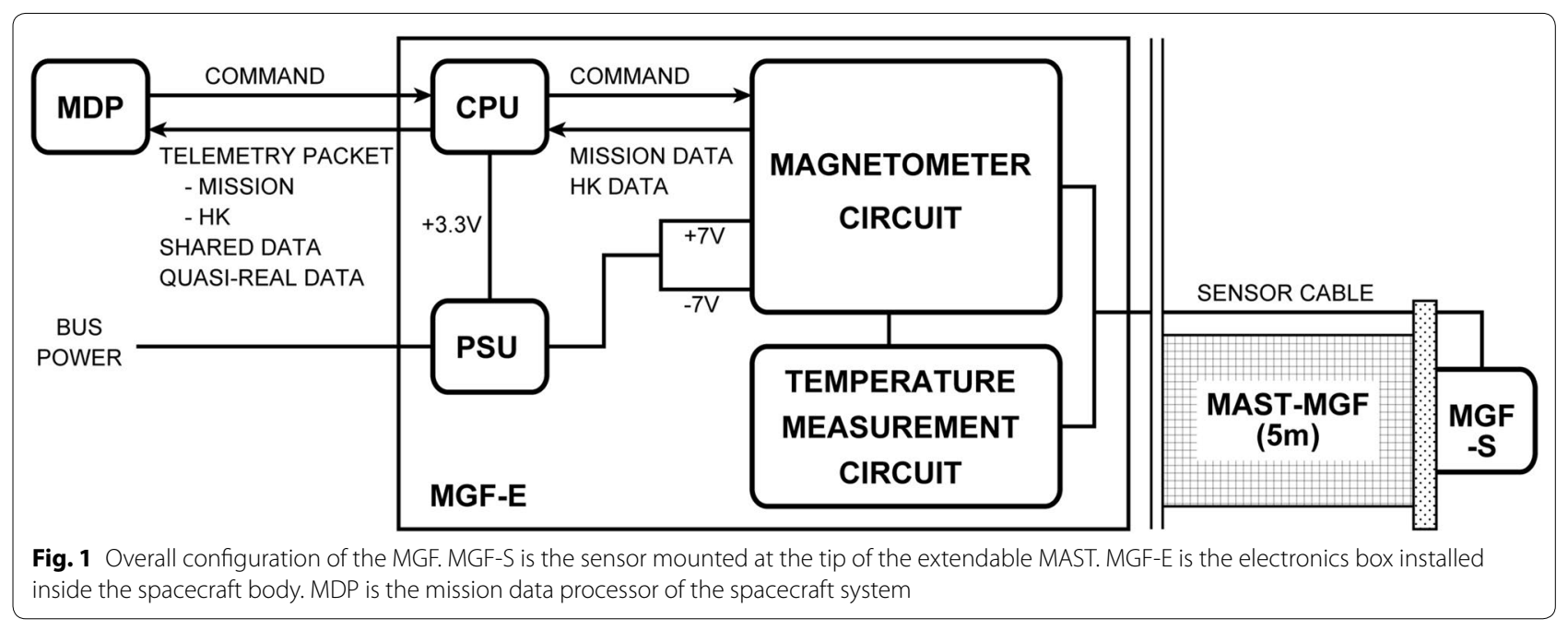


Figure 2 shows photographs of the flight model MGF-S, MGF-E, and extendable MAST in stowed configuration.

\section{Sensor (MGF-S)}

In the MGF-S unit, three sensor elements are mounted on the sensor base to measure the three orthogonal components of the magnetic field. A sensor element consists of a metal core, a driving coil, and a pickup/feedback coil. The core has an identical design to the 20-mm-diameter ring-shaped nickel-molybdenum Permalloy core for BepiColombo MMO MGF-IS. The driving coil is wound around the ring to excite the magnetic field in the core. The center axis of the pickup/feedback coil is along the diameter of the ring; this axis corresponds to the field measurement direction of the sensor element. A Pt temperature sensor is located on the back side of the sensor base. Temperature data are used to calibrate the magnetic field data.
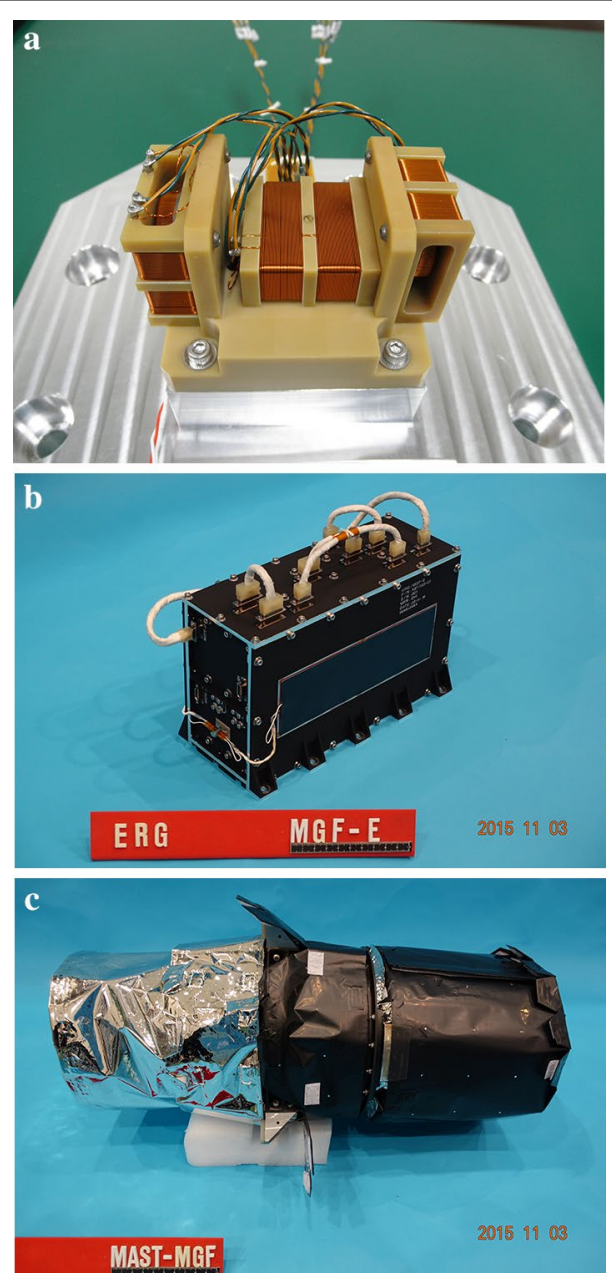

Fig. 2 Photographs of the flight models a MGF-S, b MGF-E, and c the extendable MAST in stowed configuration. The MGF-S is mounted on the MAST and is covered by a black thermal material

\section{5-m extendable MAST}

The MGF sensor is mounted on the tip of the 5-m extendable MAST to avoid magnetic interference from the spacecraft. The MAST for Arase has nearly the same design as that for Nozomi (Yamamoto and Matsuoka 1998) and is similar to those used for Geotail (Kokubun et al. 1994), Kaguya (Tsunakawa et al. 2010), and BepiColombo MMO, except for the diameter, length, and material used. The MAST was in the stowed configuration at launch, and was deployed about 1 month after launch. Arase has two MAST units, one for the MGF sensor and the other for the search-coil sensor; these were deployed simultaneously in orbit to retain the dynamic balance of the spacecraft.

\section{Circuit design}

Figure 3 shows a block diagram of the MGF magnetic field measurement function. The sensor driver circuit excites an $11 \mathrm{kHz}$ current in the drive coil wound around the sensor core. The signal amplitude detected by the sensor pickup coil at the second harmonic, $22 \mathrm{kHz}$, is proportional to the magnetic field. The $22 \mathrm{kHz}$ component is selectively amplified by bandpass filters. The phase detector outputs a voltage proportional to the amplitude of the $22 \mathrm{kHz}$ component in the pickup signal. It is integrated and produces a feedback current to the pickup coil to cancel out the external magnetic field at the sensor element. The feedback register defines the sensitivity, which is the ratio between the magnetic field and the output voltage of the integrator. Two feedback registers correspond to the two dynamic ranges, \pm 8000 and $\pm 60,000$ nT. A fieldprogrammable gate array (FPGA) switches the feedback registers and controls synchronous timing of the phase detector according to commands sent from the ground.

When magnetic cancelation at the sensor is established, the output from the integrator represents the external magnetic field. The output is converted into a 20-bit digital signal by the analog-to-digital conversion (ADC) circuit, which consists of a delta-sigma modulator and a digital filter programed in the FPGA. The FPGA stores the filtered magnetic field (mission) data in a first-in firstout (FIFO) buffer with time stamps. Sensor temperature data are obtained from a different ADC in the temperature measurement board. The FPGA transfers the mission data stored in the FIFO buffer to the CPU, together with the HK data. The HK data include the sensor temperature, parameters used to operate the magnetometer board (synchronous timing for the phase detector and range information), and Spacewire connection status.

\section{Performance of MGF}

Basic characteristics

Table 1 shows basic parameters representing the characteristics of the MGF. Two dynamic ranges, \pm 8000 and 


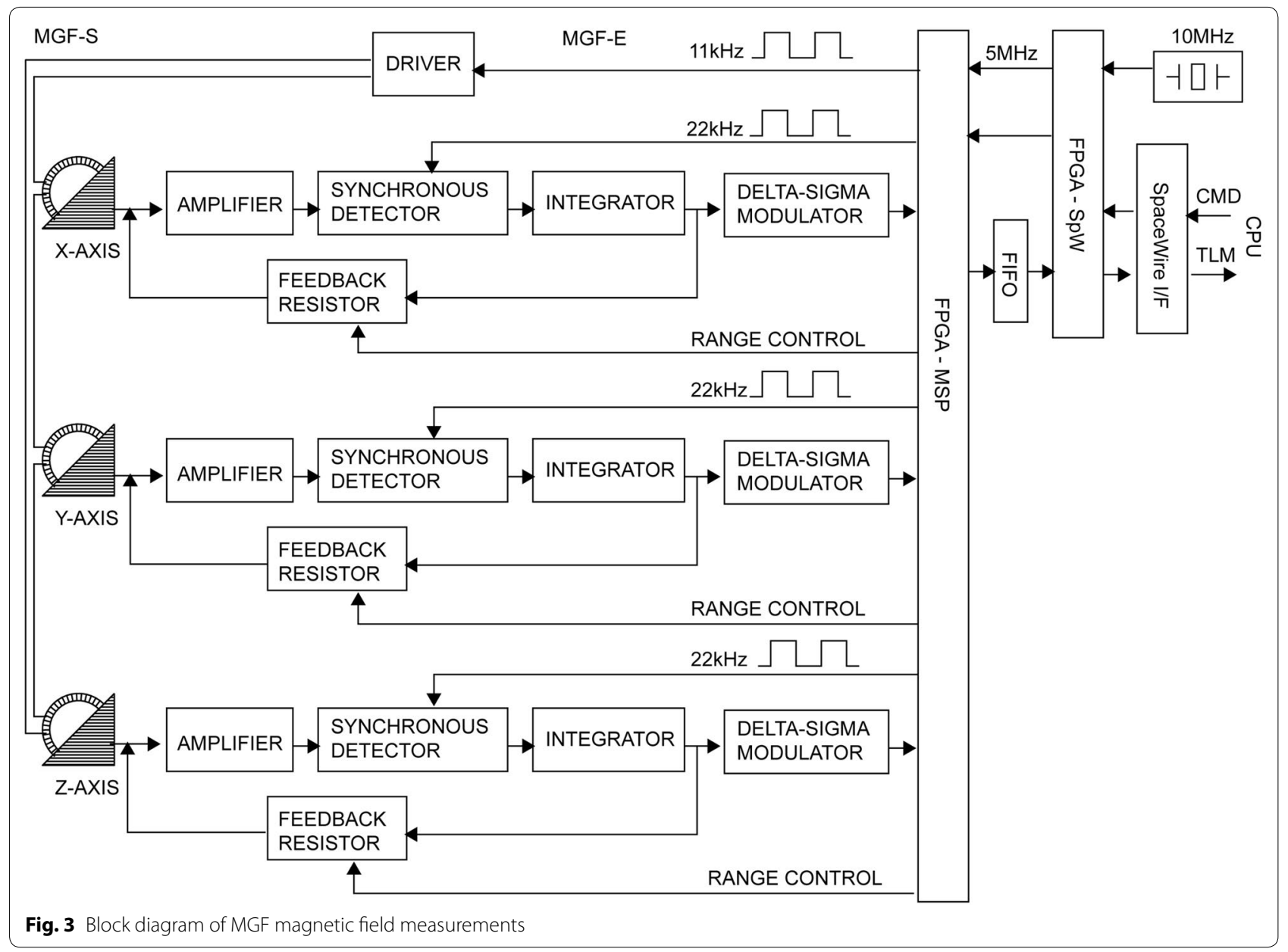

Table 1 Basic parameters of MGF for Arase (ERG)

\begin{tabular}{|c|c|c|}
\hline \multirow[t]{3}{*}{ Data sampling } & Dynamic range (nT) & $\pm 8000 \mathrm{nT} / \pm 60,000 \mathrm{nT}$ \\
\hline & Digital resolution (pT) & 15/114 (20 bits) \\
\hline & $\begin{array}{l}\text { Original sampling } \\
\text { frequency }(\mathrm{Hz})\end{array}$ & 256 \\
\hline \multirow[t]{3}{*}{ Accuracy (RT) } & Sensitivity & $\begin{array}{l}<0.06 \%( \pm 8000 \mathrm{nT} \\
\quad \text { range })\end{array}$ \\
\hline & & $\begin{array}{l}<0.10 \%( \pm 60,000 \mathrm{nT} \\
\text { range })\end{array}$ \\
\hline & Orthogonality $\left(^{\circ}\right)$ & $<0.1$ \\
\hline \multirow[t]{2}{*}{$\begin{array}{l}\text { Accuracy }(-20 \text { to } \\
\left.30^{\circ} \mathrm{C}\right)\end{array}$} & $\begin{array}{l}\text { Sensitivity change } \\
\text { from RT }\end{array}$ & $\begin{array}{l}0.016 \%( \pm 8000 \mathrm{nT} \\
\text { range })\end{array}$ \\
\hline & Offset (nT) & $\begin{array}{l}<2 \mathrm{nT}( \pm 8000 \mathrm{nT} \\
\text { range })\end{array}$ \\
\hline \multirow[t]{2}{*}{ Weight } & Sensor (g) & 120 \\
\hline & Electronics (g) & 2780 \\
\hline \multirow[t]{2}{*}{ Dimension } & Sensor (mm) & $71 \times 58 \times 41 \mathrm{H}$ \\
\hline & Electronics (mm) & $334 \times 121 \times 203 \mathrm{H}$ \\
\hline \multirow[t]{3}{*}{ Power consumption } & $+3.3 \mathrm{~V}$ & 932 mA (nominal) \\
\hline & $+7 V$ & 357 mA (nominal) \\
\hline & $-7 V$ & 130 mA (nominal) \\
\hline
\end{tabular}

$\pm 60,000 \mathrm{nT}$, are implemented for measurements at $L>2$ and $L<2$, respectively. The ADC is designed for a sampling rate of 256 vectors/s and digital resolution of 20 bits. The accuracies shown in the table are derived from ground calibration experiments, described in "Ground calibration" section.

\section{MGF performance}

Figure 4 shows the noise spectrum of the analog output when the MGF-S sensor is placed in a magnetic shielding box. The peaks at 20,50, and $60 \mathrm{~Hz}$ and higher harmonics are associated with environmental noise. Noise appearing at other frequencies is mostly attributed to the Barkhausen noise of the sensor. The properties of the noise are summarized in Table 2. In the dynamic range of $\pm 8000 \mathrm{nT}$, the typical noise intensity at $1 \mathrm{~Hz}$ is $9-10 \mathrm{pT} / \sqrt{\mathrm{Hz}}$, and the root mean square (RMS) amplitude of the noise in the frequency band from $0.1-10 \mathrm{~Hz}$ is $35-40 \mathrm{pT}$. The noise generated in the ADC part was examined by investigating the digital output with stable voltage supplied to the input. The RMS amplitude of 

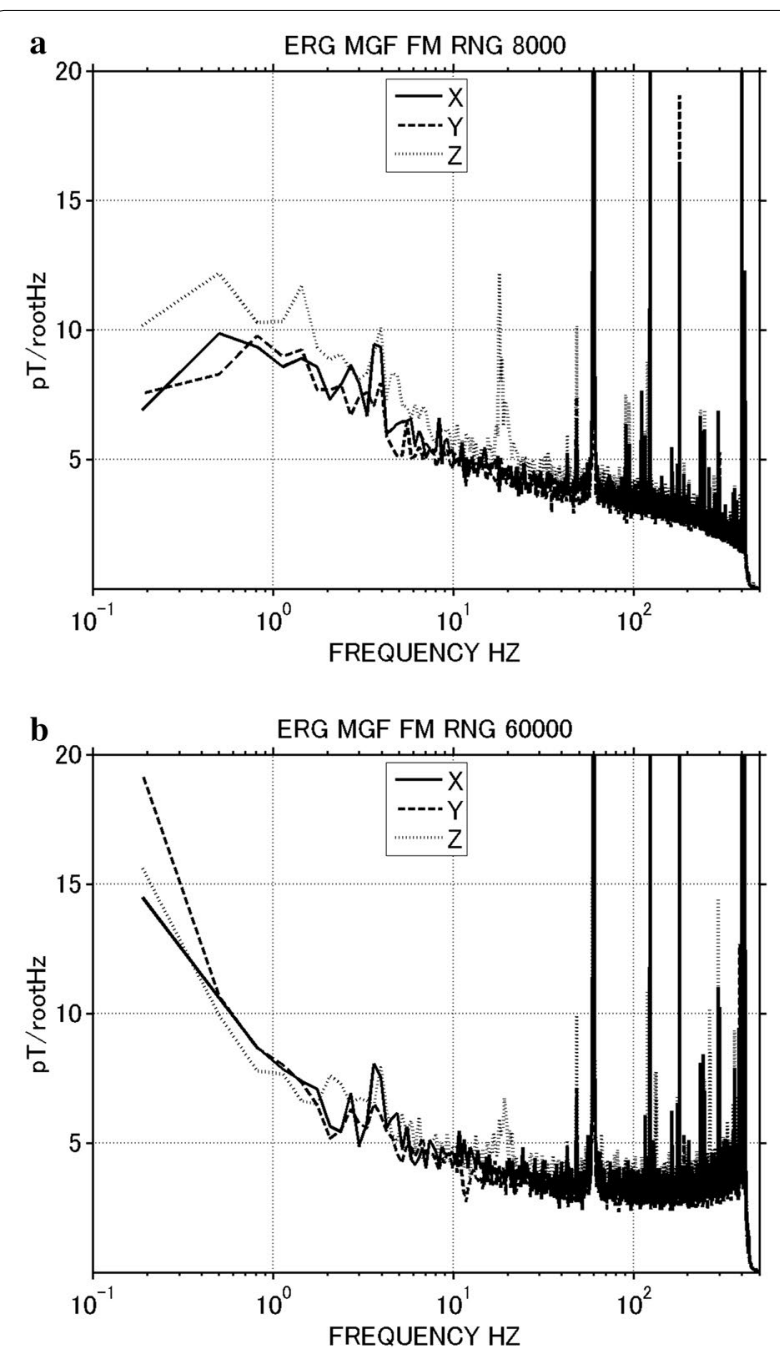

Fig. 4 Noise spectrum of the analog output obtained when the MGF-S sensor was placed in a magnetic shielding box: $\mathbf{a} \pm 8000 \mathrm{nT}$ range and $\mathbf{b} \pm 60,000 \mathrm{nT}$ range

the output was about 2 least significant bits (LSBs) corresponding to $31 \mathrm{pT}$, which is comparable to the noise generated by the analog part. These results satisfy the sensitivity requirement that the noise floor be lower than $80 \mathrm{pT} / \sqrt{\mathrm{Hz}}$ ("Requirements for the MGF" section). Similarly, in the $\pm 60,000 \mathrm{nT}$ range, the analog noise levels at 1 and $0.1-10 \mathrm{~Hz}$ RMS are $8-9 \mathrm{pT} / \sqrt{\mathrm{Hz}}$ and $32-34 \mathrm{pT}$, respectively. However, the resolution at the digital output is never better than the ADC noise intensity, 2 LSBs, which corresponds to $230 \mathrm{pT}$.

Figure 5 shows the response characteristics of the MGF analog part. The line plots show the integrator output (Fig. 3) for calibration signals applied to the sensor. The cutoff frequency $(-3 \mathrm{~dB})$ is higher than $200 \mathrm{~Hz}$ for the $\pm 8000 \mathrm{nT}$ range, and higher than $300 \mathrm{~Hz}$ for the $\pm 60,000 \mathrm{nT}$ range. In the frequency range of $1-107 \mathrm{~Hz}$
(107 Hz is the frequency nearest the cutoff of the ADC digital filter described later), phase delay at each frequency is determined with an accuracy better than 35 (20) $\mu$ s for the $\pm 8000( \pm 60,000) n$ n range. A summary of the test results is given in Table 2 .

The overall frequency characteristics of the MGF are represented by a combination of the characteristics of the analog part, $\mathrm{ADC}$, and subsequent averaging processing. Figure 6 shows the time domain window function and the corresponding frequency response of the $\mathrm{ADC}$, combined with the subsequent averaging processor. The ADC modulates the analog magnetic field data into delta-sigma $65 \mathrm{kHz}$ signals. A finite impulse response (FIR) digital filter is applied to these signals, and $256 \mathrm{~Hz}$ samples are generated. The window length of the FIR filter is twice the sampling interval, $7.8 \mathrm{~ms}$. The frequency response of the FIR filter, shown by a solid line in Fig. 6b, has a cutoff at $103.5 \mathrm{~Hz}$, far below the cutoff frequencies of the analog signals. To reduce telemetry volume, the CPU averages the $256 \mathrm{~Hz}$ samples down and resamples the data at a reduced rate. Figure $6 \mathrm{~b}$ shows the response characteristics of the digital filters for 128,64 , and $32 \mathrm{~Hz}$ sampling frequencies, with cutoff frequencies at 55,28 , and $14 \mathrm{~Hz}$, respectively; these cutoff frequencies are far below those of analog signals. Because the time stamps are defined at the center of the data window, the FIR and averaging filters do not introduce any time delay. To summarize, MGF data are characterized by the frequency responses of the digital (FIR and averaging) filters and the time delay occurring in the analog circuit.

\section{Ground calibration}

Magnetic field sensitivity and sensor orthogonality were measured in the magnetic test facility at the Japan Aerospace Exploration Agency (JAXA) Tsukuba Space Center. The experimental details and results are discussed in Teramoto et al. (2017). Sensitivity was determined with an accuracy of $0.06 \%$ ( $\pm 8000 \mathrm{nT}$ range) and the orthogonality (angles between the measurement axes) with an accuracy of $0.03^{\circ}$ (Table 1 ).

Due to deformation of the sensor by temperature variation, the sensitivity and offset depend on the sensor temperature. The sensitivity for various sensor temperatures was measured in the magnetic shielding room at JAXA's Sagamihara Campus (Hirao et al. 1985). The increase in sensor sensitivity with temperature is caused by thermal expansion of the pickup/feedback coil dimensions. Sensitivity measurement error increased as the temperature decreased due to the difficulty in keeping the sensor temperature stable at low temperatures. Nevertheless, in the temperature range from -20 to $30{ }^{\circ} \mathrm{C}$, the sensitivity variation from that at room temperature was determined 
Table 2 Frequency characteristics of MGF

\begin{tabular}{|c|c|c|c|c|c|c|}
\hline & \multicolumn{3}{|c|}{ $\pm 8000 \mathrm{nT}$ range } & \multicolumn{3}{|c|}{ $\pm 60,000 \mathrm{nT}$ range } \\
\hline & $x$ & $Y$ & $Z$ & $x$ & $Y$ & $Z$ \\
\hline \multicolumn{7}{|l|}{ Analog part } \\
\hline Noise density @ $1 \mathrm{~Hz}(\mathrm{pT} / \sqrt{\mathrm{Hz}})$ & 8.9 & 9.5 & 10.1 & 8.4 & 8.7 & 7.7 \\
\hline Noise RMS in $0.1-10 \mathrm{~Hz}(\mathrm{pT})$ & 36.6 & 34.5 & 39.7 & 33.1 & 34.1 & 32.2 \\
\hline Cutoff $(\mathrm{Hz})$ & 275 & 213 & 275 & 326 & 326 & 326 \\
\hline Time delay @ 1 Hz (msec) & 0.889 & 1.109 & 0.924 & 0.426 & 0.438 & 0.423 \\
\hline 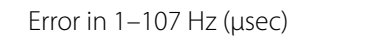 & $<35$ & $<20$ & & & & \\
\hline \multicolumn{7}{|l|}{ Digital part } \\
\hline \multicolumn{7}{|l|}{ Cutoff $(\mathrm{Hz})$} \\
\hline 256 Hz sampling & 103.5 & & & & & \\
\hline $128 \mathrm{~Hz}$ sampling & 55 & & & & & \\
\hline $64 \mathrm{~Hz}$ sampling & 28 & & & & & \\
\hline $32 \mathrm{~Hz}$ sampling & 14 & & & & & \\
\hline
\end{tabular}

within $0.016 \%$. This percentage is smaller than the determination error of the absolute sensitivity, described above $(0.06 \%$ for the $\pm 8000 \mathrm{nT}$ range and $0.1 \%$ for the $\pm 60,000$ nT range).

The measurement offset and its dependence on temperature were evaluated in the same experimental test. The offset variation was less than $2 \mathrm{nT}$ for temperatures from -20 to $30{ }^{\circ} \mathrm{C}$ (Teramoto et al. 2017), which meets the requirement of $5 \mathrm{nT}$ ("Requirements for the MGF" section). However, we must note that spacecraft magnetic cleanliness and sensor alignment, which contribute to the magnetic field offset, have yet to be addressed; these are discussed below.

\section{Spacecraft magnetic cleanliness}

To achieve precise measurements of the magnetic field, reduction in magnetic interference from the spacecraft is important. Many efforts were made to optimize the magnetic cleanliness of the Arase spacecraft. The design of the installed components and spacecraft system were examined in view of magnetic cleanliness and modified to reduce interference as needed. The magnetic fields generated by individual components were measured to confirm the effectiveness of these efforts. In this section, we present the final evaluation results for magnetic cleanliness.

The stray field around the powered-off spacecraft was measured in the magnetic shielding chamber at JAXA's Sagamihara Campus. Field vectors at $2.5-\mathrm{m}$ distance from the center of the spacecraft were measured in $30^{\circ}$ steps in elevation and $45^{\circ}$ steps in azimuth. Figure $7 \mathrm{a}$ shows the measurement results, and Fig. $7 \mathrm{~b}$ shows the superposition of the fields calculated by the measured magnetic moments of the individual components. The field intensity and its dependence on the azimuth were similar for the superposition model (Fig. 7b) and postassembly measurements (Fig. 7a). A comparison at elevation $=0^{\circ}$ and azimuth $=0^{\circ}$, which is in the same direction from the spacecraft center as MAST deployment, was most representative to check the validity of the model; measurement results indicated a field intensity of $\sim 4 \mathrm{nT}$, whereas the model predicted $\sim 3 \mathrm{nT}$. The difference is small; thus, the superposition model is considered to work well for evaluating the field around the spacecraft. The difference could be attributed to the properties of the magnetic materials used in the spacecraft, which would distort the magnetic field. The model indicates that the field intensity at the MGF-S position after MAST deployment is $0.18 \mathrm{nT}$, which is much smaller than the offset of the MGF instrument. Also, it is much lower than the required $5 \mathrm{nT}$ described in "Requirements for the MGF" section.

Time-varying fields during system-level spacecraft function tests were also measured at the $2.5-\mathrm{m}$ distance from the spacecraft center in the same magnetic shielding room. In this case, it was difficult to evaluate the noise from the spacecraft because the measured field was overwhelmed by noise believed to have originated from controllers located outside the shielding room. The noise appeared even when the spacecraft power was turned off, leading us to conclude that it must have radiated from cables connected to the controllers. For the possible worst-case scenario, in which noise from the spacecraft at the $2.5-\mathrm{m}$ distance is comparable to the environmental noise, we estimated the noise to be lower than $100 \mathrm{pT} / \sqrt{\mathrm{Hz}}$, except for a narrow-band enhancement at $7 \mathrm{~Hz}$. If we assume that the noise decreases as the cube of the distance from the spacecraft center, it is estimated to be below $9 \mathrm{pT} / \sqrt{\mathrm{Hz}}$ at the MGF-S position after MAST deployment, which is comparable to the noise of the 

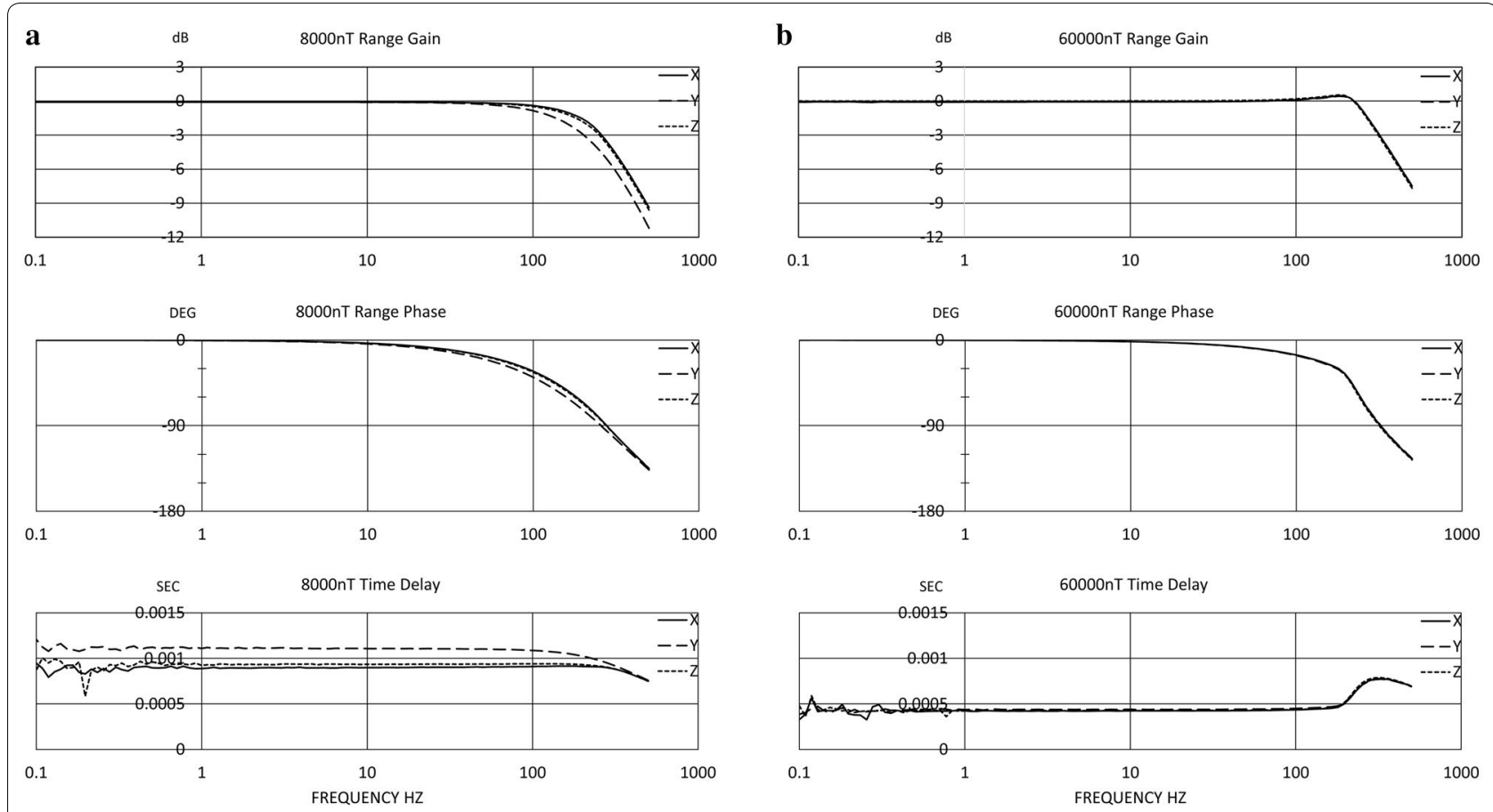

Fig. 5 Response characteristics of the MGF analog part. Response of the integrator output to the calibration signal input to the sensor. The top, middle, and bottom panels show the amplitude, phase, and time delay, respectively: $\mathbf{a} \pm 8000 \mathrm{nT}$ range and $\mathbf{b} \pm 60,000 \mathrm{nT}$ range

MGF instrument itself, as shown in Fig. 5. Moreover, it is one order of magnitude below the required noise level $(80 \mathrm{pT} / \sqrt{\mathrm{Hz}})$ described in "Requirements for the MGF" section.

From unit-level measurements and examinations of the magnetic noise prior to the system-level measurement, we determined that battery charging and the magnetic torquer generate time-varying magnetic noise over the required level. During the system-level spacecraft function test in the shielding room, noise from the spacecraft exceeded the environmental noise when the battery was fast-charging and when the magnetic torquer was in operation. Field variation generated by the battery charging current had an amplitude of $\sim 6 \mathrm{nT}$ at $2.5 \mathrm{~m}$ from the spacecraft center, corresponding to an amplitude of $\sim 0.5 \mathrm{nT}$ at the tip of the deployed MAST. Variation during torquer operation was about 70 times larger. Therefore, caution is warranted when using data acquired during fast battery charging after an eclipse and at all times when using data acquired during torquer operation, which occurs at perigee in every orbital revolution.

\section{Operation}

\section{Onboard data processing}

As shown in Fig. 3, the mission and HK data from the MGF are transferred from the FPGA on the magnetometer circuit board to the CPU by the Spacewire protocol. The CPU board processes the mission and HK data; it also edits data packets that are transferred to the MDP. The data packets are transferred to the ground via the MDP and data management component (DMC) of the spacecraft system. In addition, the CPU generates near-real-time onboard shared data. These data are written into the shared data area in the relay packet and are then circulated among the scientific instruments and transferred to other instruments and the MDP.

Table 3 shows a list of magnetic field data produced by the MGF CPU. The MGF mission telemetry packet, which comprises magnetic field data and status information necessary for data processing on the ground, has five formats that are mutually exclusive. The checkout format contains all information in the mission data from the magnetometer circuit board. With the exception of the checkout mode, the format differs with respect to the data sampling rate: $256 \mathrm{~Hz}$ (same as the original), 128, 64, or $32 \mathrm{~Hz}$. The CPU software extracts valid data from the original magnetic field data transferred from the magnetometer circuit board and averages them according to the sampling rate.

The MGF HK telemetry packet contains all of the hardware HK status from the magnetometer circuit board, software status, and decimated magnetic field data. Although the HK telemetry packet is produced every 


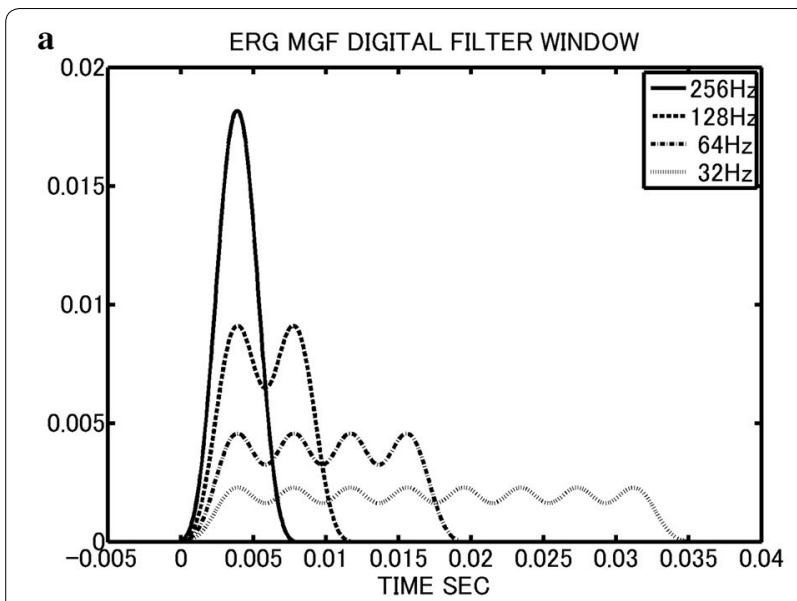

b ERG MGF DIGITAL FILTER CHARACTERISTICS

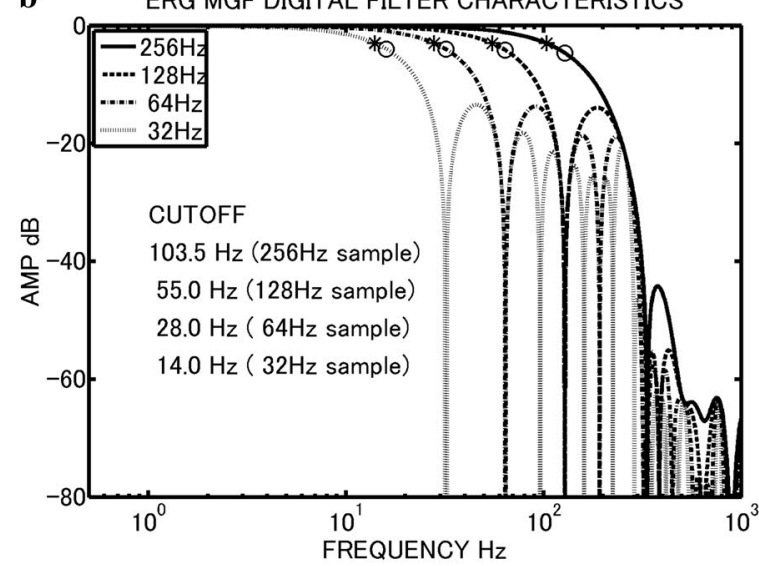

Fig. 6 a Window shape and $\mathbf{b}$ response characteristics of the digital filter in the analog-to-digital convertor (ADC) and subsequent averaging processor for the MGF. The asterisks represent the Nyquist (half of the sampling) frequencies. The circles indicate the cutoff frequencies

second, this information is often made available only at a lower rate (nominally, once every $5 \mathrm{~s}$ ) on the ground. Selected items in the HK data are compiled in spacecraft system HK packets and made available every second to ground control.

Onboard CPU software generates near-real-time and onboard shared data from the measured magnetic field data. Data are preliminarily calibrated based on the results of ground calibration experiments. Magnetic field data at spin-time resolution are included in the Space Weather data packet and transferred to the ground immediately, where they are used as a near-realtime indicator of the inner magnetosphere's condition. In contrast, shared MGF data, which have a cadence of $16 \mathrm{~Hz}$, become instantaneously available to the CPUs of other science instruments and are used for onboard data processing.

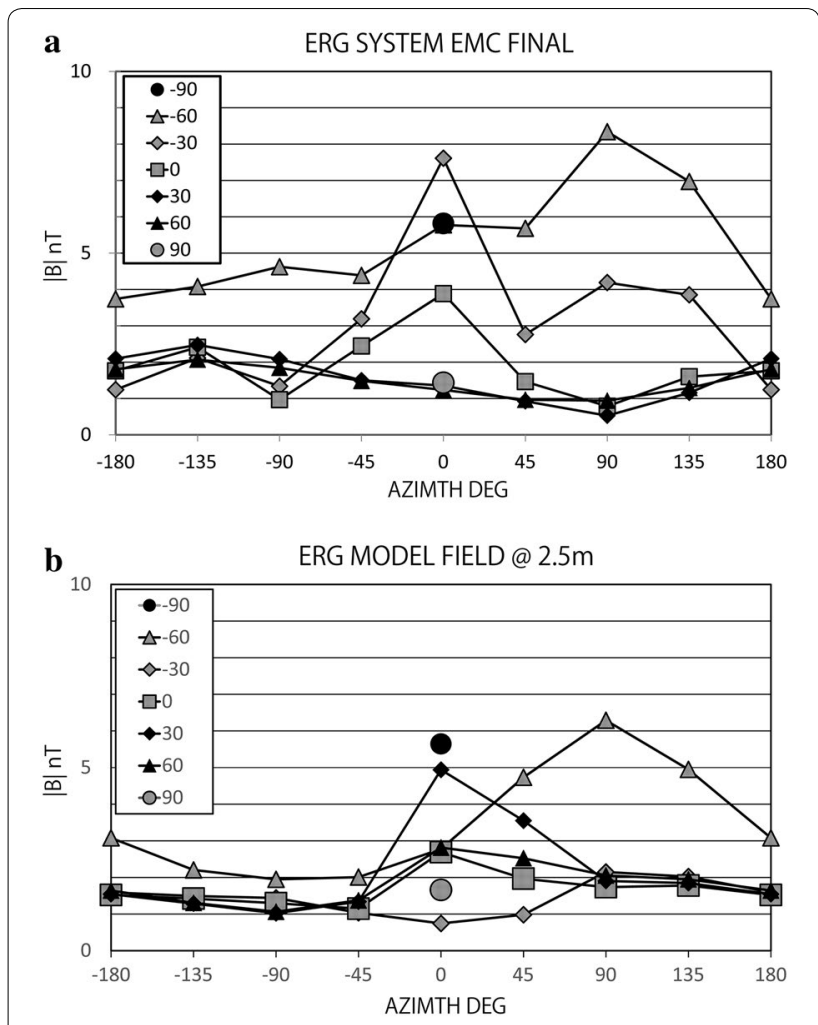

Fig. 7 Field intensity at a 2.5-m distance from the spacecraft center, measured every $30^{\circ}$ in elevation and every $45^{\circ}$ in azimuth. Individual lines correspond to different elevations: a measurement results of the magnetic field around the Arase spacecraft and $\mathbf{b}$ superposition of the fields calculated by the measured magnetic moments of the individual components

\section{Initial operation}

The initial MGF operations were carried out from January to March 2017, during which time, no sciencequality data were produced. Table 4 shows the history of the operation. The initial checkout of the MGF was performed on January 10, and all functions of the MGF were confirmed to be normal. The MAST was deployed on January 17 without incident. Autonomous control of the dynamic range by the CPU software was initiated on February 8. The CPU software was updated on March 10 to accommodate regular observation.

\section{Regular operation}

MGF data have been acquired regularly since initiation on March 13, 2017. As of the time of the preparation of this manuscript (September 2017), the sampling rates of telemetry packet data are $256 \mathrm{~Hz}$ for $L<4$ and $64 \mathrm{~Hz}$ for $L>4$. These rates were selected to cover the proton cyclotron frequency, which is about $10 \mathrm{~Hz}$ at $L=4$ and decreases with increasing $L$. 
Table 3 MGF data produced by CPU

\begin{tabular}{|c|c|c|c|c|c|}
\hline Data type & $\begin{array}{l}\text { Available on the } \\
\text { ground? }\end{array}$ & Data rate & B field resolution & Coordinate & Note \\
\hline \multirow[t]{4}{*}{ Mission } & \multirow[t]{4}{*}{ Y } & $256 \mathrm{~Hz}$ & \multirow{4}{*}{$\begin{array}{l}16 \mathrm{pT}( \pm 8000 \mathrm{nT} \text { range }) \\
125 \mathrm{pT}( \pm 60,000 \mathrm{nT} \text { range })\end{array}$} & \multirow[t]{5}{*}{ Sensor XY/Z } & \multirow[t]{5}{*}{ Uncalibrated } \\
\hline & & $128 \mathrm{~Hz}$ & & & \\
\hline & & $64 \mathrm{~Hz}$ & & & \\
\hline & & $32 \mathrm{~Hz}$ & & & \\
\hline Checkout & Y & $256 \mathrm{~Hz}$ & & & \\
\hline Shared & N & $16 \mathrm{~Hz}$ & \multirow{2}{*}{$\begin{array}{l}\text { B intensity } \times 3 \times 10^{-5} \\
\text { Or } \\
62.5 \mathrm{pT}( \pm 8000 \mathrm{nT} \text { range }) \\
4 \mathrm{nT}( \pm 60,000 \mathrm{nT} \text { range })\end{array}$} & \multirow{2}{*}{$\begin{array}{l}|B| \text { and co-angles in } \mathrm{S} / \mathrm{C} \\
\text { coordinate }\end{array}$} & \multirow[t]{2}{*}{ Coarsely calibrated } \\
\hline $\begin{array}{l}\text { Near real-time (space } \\
\text { weather) }\end{array}$ & Y & $1 /$ spin & & & \\
\hline
\end{tabular}

Table 4 Major events of MGF in the initial operation period after launch

\begin{tabular}{ll}
\hline DATE in UT (month/day, 2017) & Events \\
\hline $1 / 10$ & Initial checkout \\
$1 / 17$ & MAST deployment \\
$1 / 25$ & Test of CPU function to switch \\
& dynamic range autonomously \\
$2 / 5$ & Test of CPU function for onboard \\
& coarse calibration \\
& Onboard shared data verification \\
$2 / 8$ & Upload of preliminary parameters for \\
& onboard coarse calibration \\
& Start autonomous switching of \\
& dynamic range by CPU \\
$2 / 17$ & Start MGF operation by timeline \\
$3 / 7$ & Update parameters for onboard \\
& coarse calibration \\
$3 / 10$ & Update CPU software to adjust aver- \\
& aging process \\
$3 / 13$ & Start regular observation \\
\hline
\end{tabular}

For autonomous control of the dynamic range, the CPU software checks the peak field intensity from the three axis measurements during spacecraft rotation. To avoid unnecessary switching, the CPU changes the dynamic range only when the peak value exceeds a threshold for three successive rotations.

Figure 8 shows a plot of the magnetic field intensity measured by the MGF on March 28, 2017; the intensity results obtained using the IGRF model are included for comparison. The field intensity varied from $\sim 100 \mathrm{nT}$ at apogee to $\sim 30,000 \mathrm{nT}$ at perigee, with an orbital period of $9.5 \mathrm{~h}$. As mentioned above, the sampling rate is $256 \mathrm{~Hz}$ for $L<4$ and $64 \mathrm{~Hz}$ for the rest period. The dynamic range is $\pm 60,000 \mathrm{nT}$ for about $1 \mathrm{~h}$ at perigee and $\pm 8000 \mathrm{nT}$ for the rest. On the logarithmic scale, the measured field intensity agrees well with the IGRF model when it is larger than $1000 \mathrm{nT}$; however, differences become more pronounced when the field is weak. This means that the external field becomes more dominant as $\mathrm{L}$ increases. The difference is expected because this example was taken during the recovery phase of a storm driven by a co-rotating interaction region (CIR) in the solar wind.

Figure 9 shows an example of magnetic field measurement by the three sensor elements of the MGF. The $X$-axis is nearly along the MAST extension direction, the $Z$-axis is nearly parallel to the spacecraft $Z$-direction, and the $Y$-axis completes the right-hand coordinate. Because the spacecraft rotates about a spin axis aligned with the spacecraft's $Z$-axis, MGF $X$ and $Y$ components show a sinusoidal wave form with a spin period of about $8 \mathrm{~s}$. It is noteworthy that the $Z$-component shows a smallamplitude sinusoidal variation as well. Moreover, further inspection reveals that the amplitudes are slightly different between the $X$ and $Y$ components; this is caused by the inclination of the measurement directions of the sensor elements from the reference coordinates with respect to the spacecraft spin axis.

\section{In-orbit calibration}

To precisely measure the magnetic field, we need to accurately evaluate sensor element alignments in the spacecraft reference frame. Knowledge of the alignment is also necessary for accurate determination of the measurement offset.

The alignment is difficult to determine in ground experiments. Moreover, the alignment may change over time due to deformation of the MAST. The inclination angles can be calculated from the amplitude and phase of the sinusoidal wave forms in in-orbit data. Let us consider the simplest configuration when the directions of sensor elements $X$ and $Y$ perfectly coincide with the spacecraft $X$ and $Y$ directions, and the $Z$ element directs to $(\sin \gamma \cos \phi, \sin \gamma \sin \phi, \cos \gamma)$ in the spacecraft reference frame. The ratio of the time-varying waveform amplitudes between data of $X / Y$ elements and those of $Z$ element is $\sin \gamma$. The phase difference between data of $X$ element and those of $Z$ element is $\phi$. The relationship 


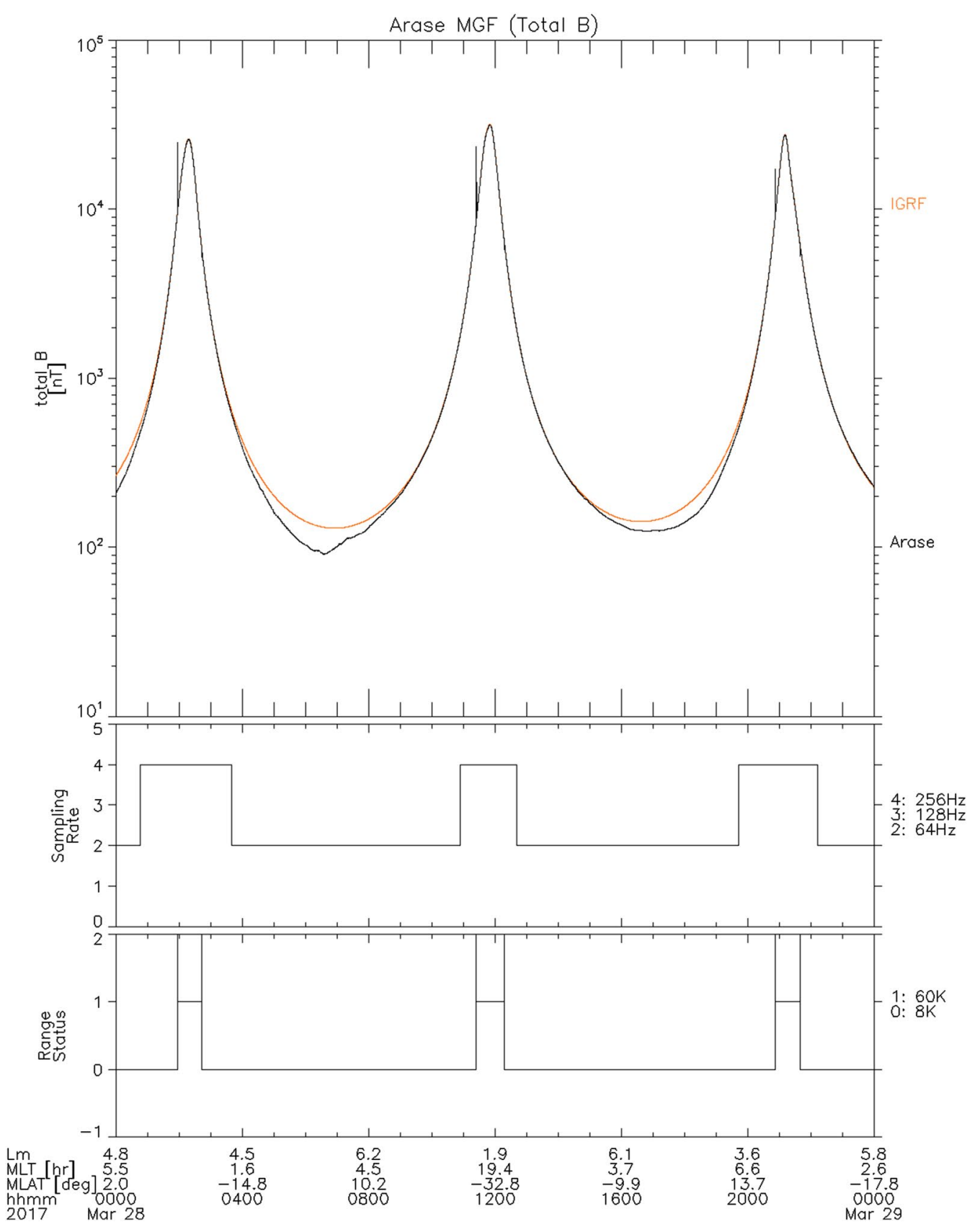

Fig. 8 Plot of the magnetic field intensity observed by the MGF on March 28, 2017, and by the International Geomagnetic Reference Field (IGRF) model. The second panel shows the sampling rate of downlinked data, and the bottom shows the dynamic range status

becomes much more complicated when the inclinations of $X$ and $Y$ elements are considered. Figure 10 shows the relationship between non-orthogonal sensor coordinates and the spacecraft reference frame, as well as the definition of misalignment angles $\alpha$ and $\beta$. We calculated the misalignment angles for every spacecraft rotation and took daily statistics of the results to determine $\alpha$ and $\beta$ for the calibration.
Figure 11 shows the distributions of the sensor misalignment angles on March 19, 2017, the same day as Fig. 9. In the $\pm 8000 \mathrm{nT}$ dynamic range, the distribution of $\alpha(\beta)$ exhibited a clear peak at $-0.89^{\circ}\left(-0.91^{\circ}\right)$ (Fig. 11a), and 73\% (88\%) of the $\alpha(\beta)$ samples were within $\pm 0.05^{\circ}$ of the peak. In the $\pm 60,000 \mathrm{nT}$ dynamic range, the distribution was broader (Fig. 11b), and 78\% (93\%) of the $\alpha(\beta)$ samples were within $\pm 0.20^{\circ}$ of the median 


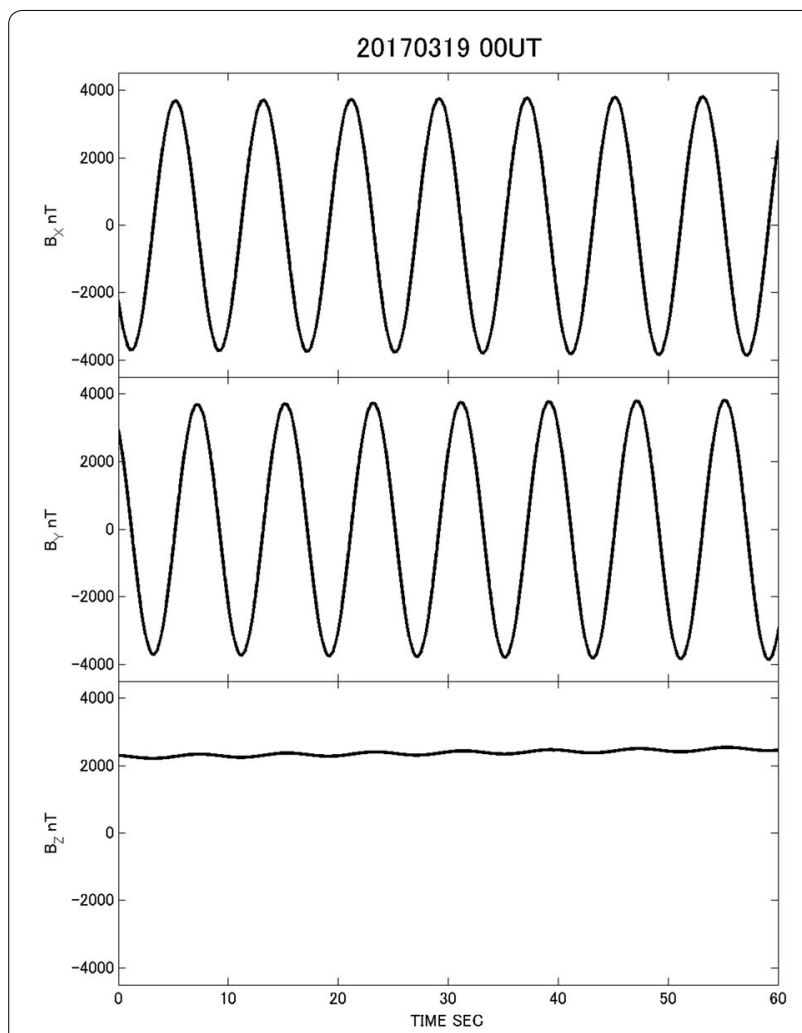

Fig. 9 Example of magnetic field measurements by the three MGF sensor elements. Data are plotted for $60 \mathrm{~s}$, starting at 00 UT on March 19,2017 . The $X$-axis is nearly along the MAST extension direction, the $Z$-axis is nearly parallel to the spacecraft $Z$-direction, and the $Y$-axis completes the right-hand coordinate

value of $-0.93^{\circ}\left(-0.30^{\circ}\right)$. We note that the field intensity changes rapidly near the perigees, where the dynamic range is nominally $\pm 60,000 \mathrm{nT}$. Time variation of the field intensity during a spin period could cause errors and broadening of the distribution of the calculated misalignment angle. The overall measurement accuracy of the magnetic field direction in inertial reference frames (geophysical coordinates) is determined by both the sensor alignment accuracy in the spacecraft reference frame and the angular accuracy of the spacecraft attitude determination. To date, we have yet to obtain full accuracy in spacecraft attitude. Therefore, the alignment accuracy in the inertial frame of reference is yet to be determined. However, if we assume a typical maximum error of the satellite attitude determination of $0.5^{\circ}$, the overall accuracy of the magnetic field direction is better than $1^{\circ}$, the requirement described in "Requirements for the MGF" section, for both the \pm 8000 and $\pm 60,000 \mathrm{nT}$ dynamic ranges. The accuracy for the $\pm 60,000 \mathrm{nT}$ range is expected to be improved by an upgraded analysis approach that considers field intensity variation.

Measurement offsets of the magnetic field in the spacecraft spin plane are evaluated using spacecraft rotation. The positive or negative shift values of the sinusoidal wave forms of the measured data are the summation of the artificial measurement offset and the spin-axis component of the natural field. The artificial measurement offsets are determined by separating these two, considering the sensor alignment. The results are consistent with those from the ground calibration test (Teramoto et al. 2017); i.e., the in-flight data offset is dominated by the MGF instrumental offset. This is reasonable, as the magnetic offset generated by the spacecraft is negligible compared with the offset by the instrument, as described in "Spacecraft magnetic cleanliness" section. We will check this point by examining the consistency between measured and model (i.e., IGRF) fields.

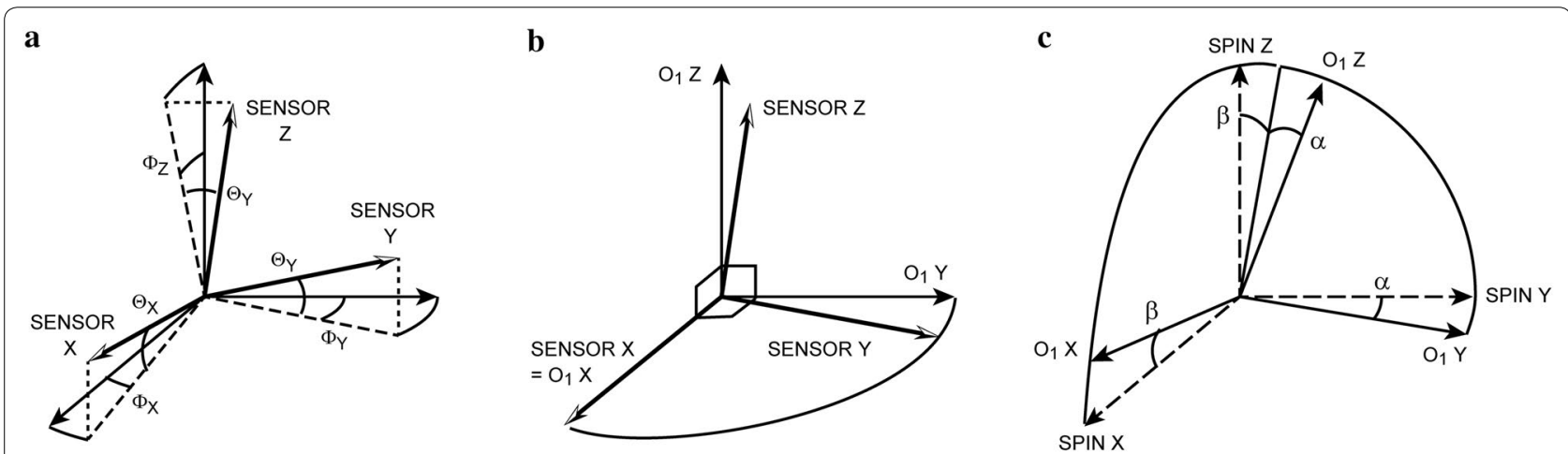

Fig. 10 Relationship between sensor non-orthogonal coordinates and the spacecraft reference frame, as well as the definitions of the misalignment angles, $a$ and $\beta$. a Angles of measurement directions in an orthogonal reference frame represented by $\Phi_{X}, \Phi_{Y}, \Phi_{Z}, \Theta_{X}, \Theta_{Y}$, and $\Theta_{Z}$ are determined in the ground calibration. $\mathbf{b} O_{1}$ is defined as an orthogonal coordinate that has the same $X$-direction and coplanar $X-Y$ with the sensor. $\mathbf{c}$ Sensor misalignment is expressed by inclination angles $a$ and $\beta$ of $O_{1}$ in the reference coordinate system with respect to the spacecraft spin axis 

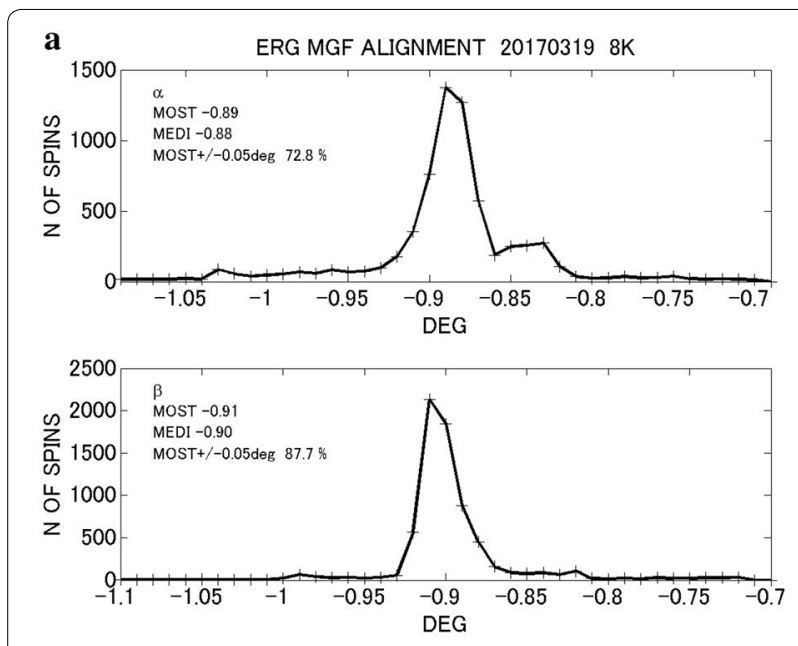

b
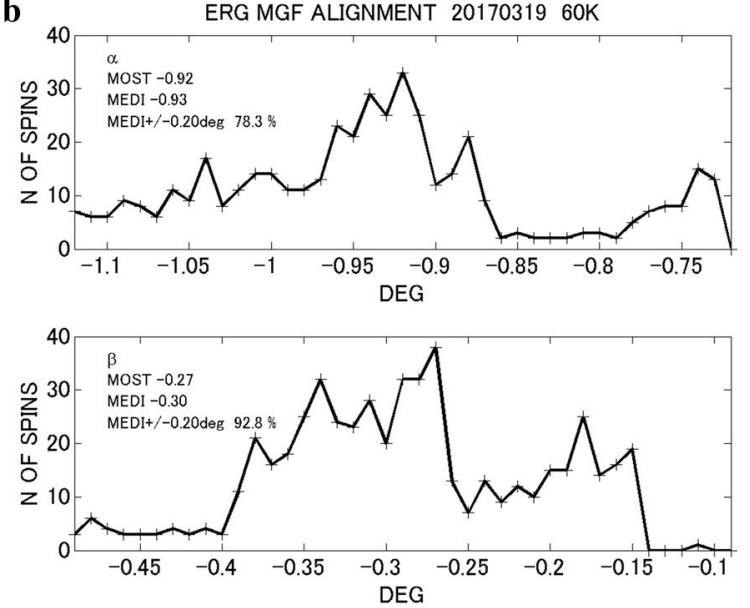

Fig. 11 Statistical results for the sensor misalignment angles $a$ and $\beta$ on March 19, 2017. a In the \pm 8000 nT dynamic range, the distribution of $a(\beta)$ has a peak at $-0.89^{\circ}\left(-0.91^{\circ}\right)$, and $72.8 \%(87.7 \%)$ of the $a(\beta)$ samples are within $\pm 0.05^{\circ}$ of the peak. $\mathbf{b}$ In the $\pm 60,000 \mathrm{nT}$ dynamic range, the median value of $a(\beta)$ is $-0.93^{\circ}\left(-0.30^{\circ}\right)$, and $78.3 \%(92.8 \%)$ of the $a(\beta)$ samples are within $\pm 0.20^{\circ}$ of the median value

\section{Data processing flow}

Arase telemetry data are stored in the Scientific Information Retrieval and Integrated Utilization System (SIRIUS) at the Institute of Space and Astronautical Science (ISAS)/JAXA. The MGF mission and HK telemetry packets are extracted from Arase telemetry data. The raw magnetic field data in the mission packets are converted into calibrated magnetic field vectors in the spacecraft reference frame using the calibration parameters.

As mentioned in "Operation" section, the sampling rate of the data in the MGF mission telemetry packet is $256,128,64$, or $32 \mathrm{~Hz}$. Data are converted into the physical magnetic field vectors in the rotating spacecraft coordinates by calibration parameters, namely sensitivity, offset, and alignment. Besides the product from the $128 \mathrm{~Hz}$ telemetry raw data, $128 \mathrm{~Hz}$ vector calibrated data are generated by averaging $256 \mathrm{~Hz}$ vector data. Similarly, $64 \mathrm{~Hz}$ vector data are generated by averaging 256 and $128 \mathrm{~Hz}$ vector data. The original and averaged vectors are despun into non-rotating spacecraft coordinates and geophysical coordinates. Spin averages are generated from the vectors in the non-rotating spacecraft coordinate and converted to vectors in the geophysical coordinates. The final products are archived as CDF files and made publicly available. The data are distributed by the ERG Science Center together with Space Physics Environment Data Analysis Software (SPEDAS) to handle the data. From March to August 2017, the sampling rate of the MGF mission telemetry data was either 256 or $64 \mathrm{~Hz}$, which means that continuous data are available at $64 \mathrm{~Hz}$ and spin-period time resolutions.

\section{Examples of in-flight measurements}

Figure 12 shows an example of MGF observation on March 27, 2017, made 1 day before the example taken during the main phase of the storm (Fig. 8). Large-amplitude ULF waves were observed, and it is clear that wave period changed over time. The wave period was $\sim 60 \mathrm{~s}$, and the waves exhibited Pc 3-4 characteristics in the first $30 \mathrm{~min}$, corresponding to $L=4.9-5.5$. Larger amplitude waves suddenly emerged at 18:25 and were present at $L=5.5-5.9$. The period then became much longer, about $400 \mathrm{~s}$, and the waves exhibited the characteristics of Pc 5 waves.

Figure 12 shows the excellent performance of the MGF in measuring ULF waves, which are major targets of the Arase mission. The wave event is presented here as a preliminary report. Detailed analyses of this and other similar events will be published elsewhere.

\section{Summary and conclusions}

Tasks including system design, performance testing, ground calibration, and onboard software coding for the MGF instrument for Arase were completed with excellent results. MGF has been under normal scientific operation since March 2017. We have used data acquired in-orbit for precise sensor alignment and offset calibration.

The well-calibrated MGF data will significantly contribute to the scientific study of radiation belts and will improve our understanding of the mechanisms underlying the production and loss of relativistic electrons. 


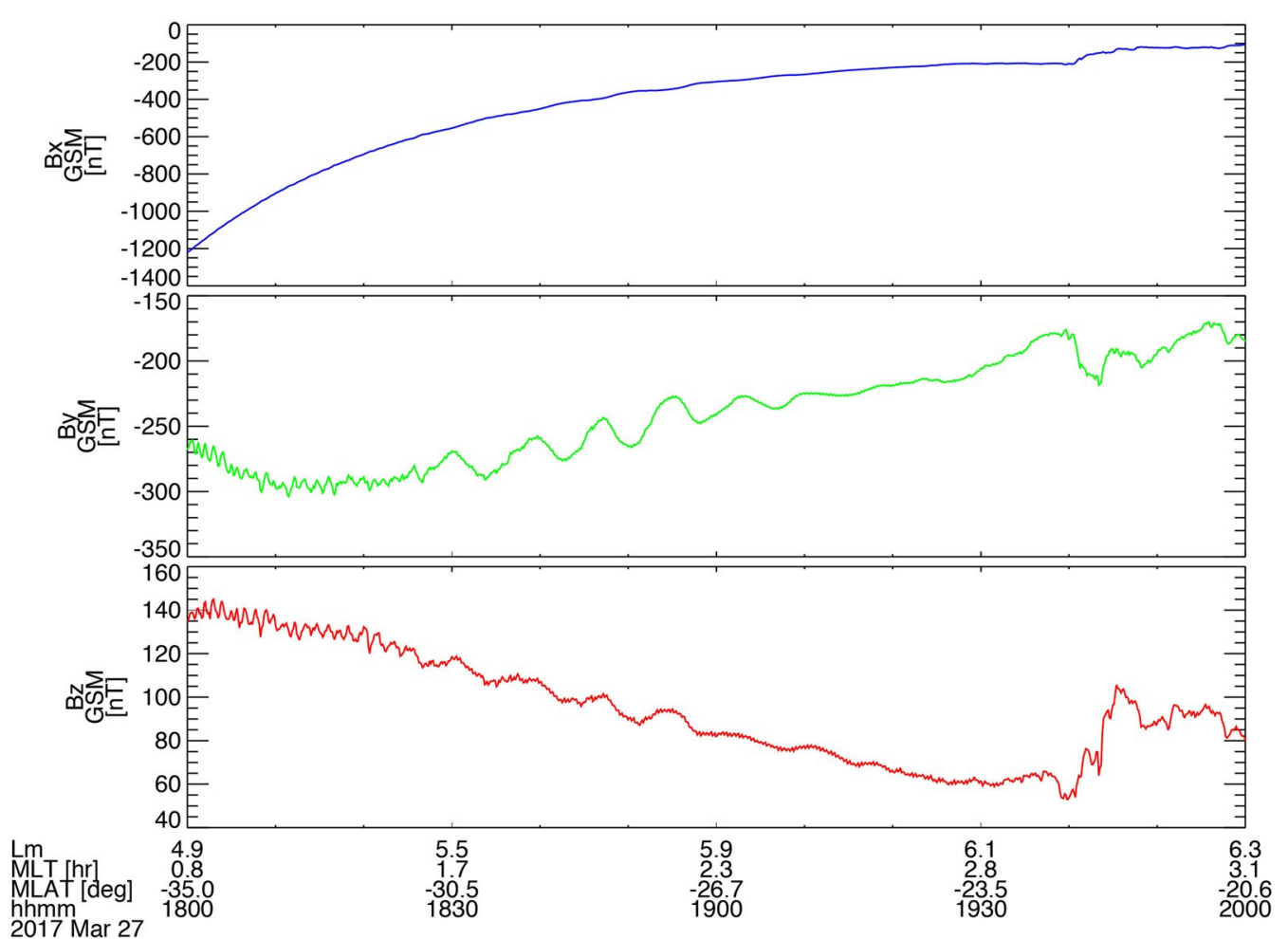

Fig. 12 Example of a MGF observation on March 27, 2017. The magnetic field vector in the Geocentric Solar Magnetospheric (GSM) coordinate system is plotted

Coordinated observation of the phenomena by multiple satellites, (e.g., the THEMIS mission, Van Allen Probes, and the Magnetospheric Multiscale (MMS) mission) and ground facilities should be promoted to further enhance the groundwork of our project (Fujimoto et al. 2012; Teramoto et al. 2016).

\section{Abbreviations}

ADC: Analog-to-digital converter; AMPTE/CCE: Active Magnetospheric Particle Tracer Explorers/Charge Composition Explorer; BBF: bursty bulk flow; CDF: Common Data Format; CIR: co-rotating interaction region; CPU: central processing unit; CRRES: Combined Release and Radiation Effects Satellite; DMC: data management component; EMIC: Electro-Magnetic lon Cyclotron; ERG: Exploration of Energization and Radiation in Geospace; FIFO: first-in firstout; FIR: finite impulse response; FPGA: field-programmable gate array; GSM: Geocentric Solar Magnetospheric; HEP: high-energy electron experiments; HK: House Keeping; IGRF: International Geomagnetic Reference Field; ISAS: Institute of Space and Astronautical Science; ISEE: Institute for Space-Earth Environment Research; JAXA: Japan Aerospace Exploration Agency; LSB: least significant bit; MAST: extendable MAST; MDP: mission data processor; MGF: Magnetic Field Experiment; MGF-E: MGF electronic box; MGF-I: MGF Inboard (BepiColombo MMO); MGF-S: MGF sensor; MMO: Mercury Magnetospheric Orbiter; MMS: Magnetospheric Multiscale; PSU: power supply unit; RMS: root mean square; RT: room temperature; SIRIUS: Scientific Information Retrieval and Integrated Utilization System; SPEDAS: Space Physics Environment Data Analysis Software; THEMIS: Time History of Events and Macroscale Interactions during Substorms; ULF: ultra-low frequency; XEP: extremely high-energy electron experiments.

\section{Authors' contributions}

AM is PI of Arase MGF. MT works for the MGF hardware test, final MGF data archive and analysis. RN works for the MGF hardware test and data analysis. MN works for the MGF data evaluation and analysis. AF works for the initial MGF planning, hardware design and data analysis. YT works for the MGF hardware development and data analysis. MS works for the MGF hardware development. TN works for the MGF data application to the space-weather forecasting. KS works for the coordination of studies by the MGF data and by ground facilities. YO works for the MGF data analysis. YM is the Arase project scientist. MM works for the EMC activities of the Arase spacecraft. TT is the Arase mission manager. IS is the Arase project manager. All authors read and approved the final manuscript.

\section{Author details}

${ }^{1}$ Institute of Space and Astronautical Science, Japan Aerospace Exploration Agency, 3-1-1, Yoshinodai, Chuo-ku, Sagamihara, Kanagawa 252-5210, Japan. ${ }^{2}$ Institute for Space-Earth Environmental Research, Nagoya University, Furo-cho, Chikusa-ku, Nagoya 464-8601, Japan. ${ }^{3}$ Environmental Test Technology Unit, Japan Aerospace Exploration Agency, 2-1-1 Sengen, Tsukuba, Ibaraki 305-8505, Japan. ${ }^{4}$ Data Analysis Center for Geomagnetism and Space Magnetism, Graduate School of Science, Kyoto University, Kitashirakawa-Oiwake Cho, Sakyo-ku, Kyoto 606-8502, Japan. ${ }^{5}$ International Center for Space Weather Science and Education, Kyushu University, 744, Motooka, Nishi-ku, Fukuoka 819-0395, Japan. ${ }^{6}$ National Institute of Polar Research, 10-3, Midori-cho, Tachikawa-shi, Tokyo 190-8518, Japan. ${ }^{7}$ National Institute of Technology, Kagoshima College, 1460-1 Shinkou Hayato-cho, Kirishima City, Kagoshima 899-5193, Japan. ${ }^{8}$ National Institute of Information and Communications Technology, 4-2-1, Nukui-kitamachi, Koganei, Tokyo 184-8795, Japan. ${ }^{9}$ Department of Engineering Science, Faculty of Engineering, Osaka ElectroCommunication University, 18-8 Hatsucho, Neyagawa, Osaka 572-8530, Japan. 


\section{Acknowledgements}

The authors wish to express their sincere thanks to all of the ERG (Arase) project team members. We are also grateful for the manufacturers of MGF, Sumitomo Heavy Industries, Ltd., and Tierra Tecnica Corporation, as well as the manufacturer of the extendable MAST, NIPPI Corporation. The MGF calibration test was supported by the Environment Test Technology Unit of JAXA. The magnetic cleanliness activities were greatly supported by Dr. Mio Murashima. Ayako Matsuoka gratefully acknowledges valuable comments from Dr. Tateo Goka and Prof. Susumu Kokubun at MGF review meetings.

\section{Competing interests}

The authors declare that they have no competing interests.

\section{Availability of data and materials}

The ERG (Arase) MGF level-2 data will be available in the data server at the ERG Science Center operated by ISAS/JAXA and ISEE/Nagoya University. (http:// ergsc.isee.nagoya-u.ac.jp/)

\section{Ethics approval and consent to participate}

Not applicable.

\section{Funding}

The Arase project is funded by ISAS/JAXA.

\section{Publisher's Note}

Springer Nature remains neutral with regard to jurisdictional claims in published maps and institutional affiliations.

Received: 20 September 2017 Accepted: 7 February 2018 Published online: 14 March 2018

\section{References}

Ali AF, Elkington SR, Tu W, Ozeke LG, Chan AA, Friedel RHW (2015) Magnetic field power spectra and magnetic radial diffusion coefficients using CRRES magnetometer data. J Geophys Res Space Phys 120:973-995. https://doi.org/10.1002/2014JA020419

Anderson BJ (1994) An overview of spacecraft observations of $10 \mathrm{~s}$ to $600 \mathrm{~s}$ period magnetic pulsations in the earth's magnetosphere. In: Engebretson MJ, Takahashi K, Scholer M (eds) Solar wind sources of magnetospheric ultra-low-frequency waves. American Geophysical Union, Washington, D.C., pp 25-43. https://doi.org/10.1029/gm081p0025

Anderson BJ, Engebretson MJ, Rounds SP, Zanetti LJ, Potemra TA (1990) A statistical study of Pc 3-5 pulsations observed by the AMPTE/CCE Magnetic Fields Experiment, 1. Occurrence distributions. J Geophys Res Space Phys 95:10495-10523. https://doi.org/10.1029/JA095iA07p10495

Anderson BJ, Erlandson RE, Zanetti LJ (1992a) A statistical study of PC 1-2 magnetic pulsations in the equatorial magnetosphere: 1. Equatorial occurrence distributions. J Geophys Res Space Phys 97:3075-3088. https://doi. org/10.1029/91ja02706

Anderson BJ, Erlandson RE, Zanetti LJ (1992b) A statistical study of PC 1-2 magnetic pulsations in the equatorial magnetosphere: 2 . Wave properties. J Geophys Res Space Phys 97:3089-3101. https://doi. org/10.1029/91ja02697

Angelopoulos V et al (1992) Bursty bulk flows in the inner central plasma sheet. J Geophys Res Space Phys 97:4027-4039. https://doi. org/10.1029/91JA02701

Baumjohann W et al (2006) The magnetosphere of Mercury and its solar wind environment: open issues and scientific questions. Adv Space Res 38:604-609. https://doi.org/10.1016/j.asr.2005.05.117

Baumjohann W et al (2010) Magnetic field investigation of Mercury's magnetosphere and the inner heliosphere by MMO/MGF. Planet Space Sci 58:279-286. https://doi.org/10.1016/j.pss.2008.05.019

Cummings WD, Barfield JN, Coleman PJ (1968) Magnetospheric substorms observed at the synchronous orbit. J Geophys Res 73:6687-6698. https:// doi.org/10.1029/JA073i021p06687
Delcourt DC (2002) Particle acceleration by inductive electric fields in the inner magnetosphere. J Atmos Solar Terr Phys 64:551-559. https://doi. org/10.1016/S1364-6826(02)00012-3

Elkington SR, Hudson MK, Chan AA (1999) Acceleration of relativistic electrons via drift-resonant interaction with toroidal-mode PC-5 ULF oscillations. Geophys Res Lett 26:3273-3276. https://doi.org/10.1029/1999GL003659

Fujimoto A, Miyoshi Y, Matsuoka A (2012) Science output from Pc 5 pulsation study by the ERG spacecraft. Trans Jpn Soc Aeronaut Space Sci Aerosp Technol Jpn 10:Tr_11-Tr_15. https://doi.org/10.2322/tastj.10.tr_11

Fukunishi $\mathrm{H}$ et al (1990) Magnetic field observations on the Akebono (EXOS-D) satellite. J Geomagn Geoelectr 42:385-409. https://doi.org/10.5636/ jgg.42.385

Gordon D, Brown R (1972) Recent advances in fluxgate magnetometry. IEEE Trans Magn 8:76-82. https://doi.org/10.1109/TMAG.1972.1067268

Hirao K, Tsuruda K, Aoyama I, Saito T (1985) Large spherical magnetic shield room. J Geomagn Geoelectr 37:581-588. https://doi.org/10.5636/ jgg.37.581

Horne RB, Thorne RM, Glauert SA, Albert JM, Meredith NP, Anderson RR (2005a) Timescale for radiation belt electron acceleration by whistler mode chorus waves. J Geophys Res Space Phys. https://doi. org/10.1029/2004ja010811

Horne RB, Thorne RM, Shprits YY, Meredith NP et al (2005b) Wave acceleration of electrons in the Van Allen radiation belts. Nature 437:227-230

Jordanova VK, Boonsiriseth A, Thorne RM, Dotan Y (2003) Ring current asymmetry from global simulations using a high-resolution electric field model. J Geophys Res Space Phys. https://doi.org/10.1029/2003ja009993

Jordanova VK et al (2006) Kinetic simulations of ring current evolution during the Geospace Environment Modeling challenge events. J Geophys Res Space Phys. https://doi.org/10.1029/2006ja011644

Keika K, Takahashi K, Ukhorskiy AY, Miyoshi Y (2013) Global characteristics of electromagnetic ion cyclotron waves: occurrence rate and its storm dependence. J Geophys Res Space Phys 118:4135-4150. https://doi. org/10.1002/jgra.50385

Kersten T, Horne RB, Glauert SA, Meredith NP, Fraser BJ, Grew RS (2014) Electron losses from the radiation belts caused by EMIC waves. J Geophys Res Space Phys 119:8820-8837. https://doi.org/10.1002/2014ja020366

Kokubun S, Yamamoto T, Acuna MH, Hayashi K, Shiokawa K, Kawano H (1994) The GEOTAIL magnetic field experiment. J Geomag Geoelectr 46:7-21

Liu WW, Rostoker G, Baker DN (1999) Internal acceleration of relativistic electrons by large-amplitude ULF pulsations. J Geophys Res Space Phys 104:17391-17407. https://doi.org/10.1029/1999ja900168

Lui ATY (1991) A synthesis of magnetospheric substorm models. J Geophys Res Space Phys 96:1849-1856. https://doi.org/10.1029/90JA02430

Matsuoka A, Shinohara M, Tanaka Y, Fujimoto A, Iguchi K (2013) Development of fluxgate magnetometers and applications to the space science missions. In: Oyama K-I, Cheng CZ (eds) An introduction to space instrumentation. Terra Scientific Publishing Company, Tokyo, pp 217-225. https:// doi.org/10.5047/aisi.021

McPherron RL, Chu X (2016) Relation of the auroral substorm to the substorm current wedge. Geosci Lett 3:12. https://doi.org/10.1186/ s40562-016-0044-5

Min K, Lee J, Keika K, Li W (2012) Global distribution of EMIC waves derived from THEMIS observations. J Geophys Res Space Phys 117:A05219. https://doi.org/10.1029/2012ja017515

Miyoshi Y, Morioka A, Misawa H, Obara T, Nagai T, Kasahara Y (2003) Rebuilding process of the outer radiation belt during the 3 November 1993 magnetic storm: NOAA and Exos-D observations. J Geophys Res Space Phys 108:SMP 3-1-SMP 3-15. https://doi.org/10.1029/2001ja007542

Miyoshi Y, Sakaguchi K, Shiokawa K, Evans D, Albert J, Connors M, Jordanova $\checkmark$ (2008) Precipitation of radiation belt electrons by EMIC waves, observed from ground and space. Geophys Res Lett. https://doi. org/10.1029/2008gl035727

Miyoshi $Y$ et al (2013) The energization and radiation in geospace (ERG) project. In: Summers D, Mann IR, Baker DN, Schulz M (eds) Dynamics of the earth's radiation belts and inner magnetosphere. American Geophysical Union, Washington, D.C., pp 103-116. https://doi. org/10.1029/2012gm001304

Miyoshi Y et al (2017) Geospace exploration project: Arase (ERG). J. Phys.: Conf. Series 869. https://doi.org/10.1088/1742-6596/869/1/012095 
Nomura R, Shiokawa K, Sakaguchi K, Otsuka Y, Connors M (2012) Polarization of PC1/EMIC waves and related proton auroras observed at subauroral latitudes. J Geophys Res Space Phys. https://doi.org/10.1029/2011ja017241

Nomura R et al (2016) Pulsating proton aurora caused by rising tone PC1 waves. J Geophys Res Space Phys 121:1608-1618. https://doi. org/10.1002/2015JA021681

Reeves GD et al (2013) Electron acceleration in the heart of the van allen radiation belts. Science 341:991-994. https://doi.org/10.1126/science.1237743

Sakaguchi K et al (2013) Akebono observations of EMIC waves in the slot region of the radiation belts. Geophys Res Lett 40:5587-5591. https://doi. org/10.1002/2013gl058258

Shprits YY, Subbotin DA, Meredith NP, Elkington SR (2008) Review of modeling of losses and sources of relativistic electrons in the outer radiation belt II: local acceleration and loss. J Atmos Solar Terr Phys 70:1694-1713. https:// doi.org/10.1016/j.jastp.2008.06.014

Su Z et al. (2015) Ultra-low-frequency wave-driven diffusion of radiation belt relativistic electrons 6:10096. https://doi.org/10.1038/ ncomms10096. http://dharmasastra.live.cf.private.springer.com/articles/ ncomms10096\#supplementary-information

Summers D, Thorne RM (2003) Relativistic electron pitch-angle scattering by electromagnetic ion cyclotron waves during geomagnetic storms. J Geophys Res Space Phys. https://doi.org/10.1029/2002ja009489
Takada T et al (2006) Do BBFs contribute to inner magnetosphere dipolarizations: concurrent cluster and double star observations. Geophys Res Lett. https://doi.org/10.1029/2006gl027440

Teramoto M, Nishitani N, Nishimura Y, Nagatsuma T (2016) Latitudinal dependence on the frequency of Pi2 pulsations near the plasmapause using THEMIS satellites and Asian-Oceanian SuperDARN radars. Earth Planets Space 68:22. https://doi.org/10.1186/s40623-016-0397-1

Teramoto M, Matsuoka A, Nomura R (2017) Ground calibration experiments of Magnetic field experiment on the ERG satellite (Japanese). JAXA Research and Development Memorandum JAXA-RM-16-003

Thorne RM, Ni B, Tao X, Horne RB, Meredith NP (2010) Scattering by chorus waves as the dominant cause of diffuse auroral precipitation. Nature 467:943-946

Tsunakawa $\mathrm{H}$ et al (2010) Lunar magnetic field observation and initial global mapping of lunar magnetic anomalies by MAP-LMAG onboard SELENE (Kaguya). Space Sci Rev 154:219-251. https://doi.org/10.1007/ s11214-010-9652-0

Yamamoto T, Matsuoka A (1998) PLANET-B magnetic fields investigation. Earth Planets Space 50:189-194. https://doi.org/10.1186/bf03352102

\section{Submit your manuscript to a SpringerOpen ${ }^{\circ}$ journal and benefit from:}

- Convenient online submission

- Rigorous peer review

- Open access: articles freely available online

- High visibility within the field

- Retaining the copyright to your article

Submit your next manuscript at springeropen.com 